\title{
Pluripotent stem cells as source of dendritic cells for immune therapy
}

\author{
Satoru Senju • Shinya Hirata $\cdot$ Yutaka Motomura • \\ Daiki Fukuma - Yusuke Matsunaga - Satoshi Fukushima • \\ Hidetake Matsuyoshi $\cdot$ Yasuharu Nishimura
}

Received: 29 October 2009/Revised: 24 November 2009/ Accepted: 26 November 2009/Published online: 13 February 2010

(C) The Japanese Society of Hematology 2010

\begin{abstract}
Dendritic cells (DC) are the most potent antigen-presenting cells. In vivo transfer of antigen-bearing $\mathrm{DC}$ has proven efficient in priming $\mathrm{T}$ cell responses specific to the antigen. DC-based cellular vaccination is now regarded as a powerful means for immunotherapy, especially for anti-cancer immunotherapy. Clinical trials of therapy with DC pulsed with peptide antigens or genetically modified to present antigens are currently carried out in many institutions. In addition, antigen-specific negative regulation of immune response by $\mathrm{DC}$ is considered to be a promising approach for treatments of autoimmune diseases and also for regulation of allo-reactive immune response causing graft rejection and GVHD in transplantation medicine. DC for transfer therapy are now generated by in vitro differentiation of peripheral blood monocytes of the patients. However, there is a limitation in the number of available monocytes, and the DC-differentiation potential of monocytes varies depending on the blood donor. Embryonic stem (ES) cells possess both pluripotency and infinite propagation capacity. We consider ES cells to be an ideal source for DC to be used in immunotherapy. Several groups, including us, have developed methods to generate
\end{abstract}

S. Senju $(\bowtie) \cdot$ S. Hirata $\cdot$ Y. Motomura $\cdot$ D. Fukuma

Y. Matsunaga - S. Fukushima - H. Matsuyoshi - Y. Nishimura

Department of Immunogenetics, Graduate School

of Medical Sciences, Kumamoto University, 1-1-1 Honjo,

Kumamoto 860-8556, Japan

e-mail: senjusat@gpo.kumamoto-u.ac.jp

S. Senju

Japan Science and Technology Agency, CREST, Tokyo, Japan

S. Senju

iPS Cell Research Laboratory, Division of Stem Cell Research, Institute of Embryology and Genetics, Kumamoto University,

Kumamoto, Japan
DC from ES cells. This review introduces the studies on generation, characterization, and genetic modification of DC derived from ES cells or induced pluripotent stem (iPS) cells. The issues to be resolved before clinical application of pluripotent stem cell-derived DC will also be discussed.

Keywords Dendritic cells - Embryonic stem cells · iPS cells · Cell therapy

\section{Introduction}

Dendritic cells (DC) are the most potent antigen-presenting cells responsible for priming of naive $T$ cells in the immune response. DC are also involved in the maintenance of immunologic self-tolerance in the periphery, inducing regulatory T cells or anergy of autoreactive T cells. We can say that DC physiologically play a central role in immune-regulation. Manipulation of functions of DC by genetic modification and in vivo transfer of DC with modified property is considered a promising means to therapeutically manipulate immune responses in an antigen-specific manner.

Embryonic stem (ES) cells are characterized by pluripotency and infinite propagation capacity. Non-virusmediated methods for gene transfer, including targeted gene integration and procedures for isolation of appropriate transfectant cell clones, have been established for ES cells. Genetic modification of ES cells and their subsequent in vitro differentiation to DC would be an attractive strategy for genetic manipulation of DC and for analysis of gene functions in DC.

For hematopoietic differentiation of ES cells in vitro, embryoid body (EB)-mediated methods and the feeder cellcoculture method have been established. OP9 is a bone marrow stromal cell line that originated from macrophage 
colony stimulating factor-defective op/op mouse, and generation of various hematopoietic cells from ES cells using OP9 cells as feeder cells has been reported, including granulocytes, erythrocytes, and B lymphocytes [1]. The method has been applied to a number of molecular and cellular analyses for investigations of hematopoiesis.

We and others developed methods to generated DC from mouse ES cells, and the functions of ES cell-derived DC (esDC or ES-DC), including stimulation of allogeneic $\mathrm{T}$ cells, processing and presentation of antigenic proteins, and migration upon in vivo transfer, were comparable to those of DC generated in vitro from bone marrow cells $[2,3]$. We have also established a strategy for the genetic modification of mouse ES-DC. Expression vectors were introduced into ES cells by electroporation and subsequently the transfectant ES cell clones were induced to differentiate to ES-DC. The studies using mice have demonstrated that in vivo transfer of genetically engineered mouse ES-DC is very effective for modulation of immune responses both positively and negatively. It is possible to induce anti-cancer immunity [4-9] and prevent autoimmune disease $[10,11]$ in mouse models with genetically engineered ES-DC. Looking toward the future clinical application of ES-DC technology, a method was developed to generate ES-DC also from human ES cells [12].

\section{Generation of dendritic cells from mouse ES cells}

\subsection{Generation of dendritic cells from mouse ES cells by embryoid body-mediated hematopoietic differentiation}

In 2000, Fairchild and his colleagues reported the first study on the generation of functional DC from mouse ES cells [2]. They used ESF116 mouse ES cell line derived from a $\mathrm{CBA} / \mathrm{Ca}$ blastocyst. In their method, at first, EB were formed, and subsequently differentiation of DC was induced by addition of specific cytokines.

At the initiation of the differentiation, ES cells were grown in suspension to allow EB formation. After culture for 14 days, EB were cultured in medium supplemented with granulocyte/macrophage colony stimulating factor (GM-CSF) and interleukin (IL)-3. Fairchild and colleagues observed that the combination of the cytokines uniquely supported the development of cells with an appearance suggestive of DC within 4 days of culture. These cells expanded rapidly over the following 7-10 days to form lightly adherent clusters, reminiscent of those observed in cultures of immature DC derived from bone marrow cells. Cells released from these clusters seeded uncolonised areas of the dish and displayed typical dendritic morphology, and they were designated as esDC.
Flowcytometric analysis showed that esDC expressed CD11c, MHC class II, CD40, CD54, CD80, and CD86. On the other hand, expression of CD8 and Dec-205 (CD205) was not observed, suggesting esDC were of myeloid lineage. RT-PCR analysis revealed that esDC expressed mRNA for IL-20, IL-18, and IL-1 $\beta$ converting enzyme (ICE). They possessed a potent capacity to process a protein antigen, hen egg lysozyme (HEL), and present the resultant epitope in the context of $\mathrm{H}-2 \mathrm{E}^{\mathrm{k}}$, shown by the response of co-cultured antigen-specific $\mathrm{T}$ cell hybridoma cells. Upon co-culture with allogeneic T cells, esDC stimulated the $\mathrm{T}$ cells to proliferate vigorously, indicating strong T cell-stimulating capacity of esDC. Collectively, the ES cell-derived cells with DC-like morphology were fully functional in antigen-presentation and T cell-stimulation.

2.2 Generation of dendritic cells from mouse ES cells by aid of OP9-coculture-based hematopoietic differentiation

We established a method to generate DC from mouse ES cells using OP9 stromal cells and our protocol is shown in Fig. 1 [3]. Mouse ES cells, maintained on primary mouse embryonic fibroblast feeder layers in the presence of leukemia inhibitory factor (LIF), were transferred onto the OP9 cell layers and cultured in $\alpha$ MEM medium containing fetal calf serum without exogenous cytokines. ES cell colonies changed their morphology from undifferentiated dome-like to a differentiated flattened one in 4-5 days (Fig. 2a, b). To induce differentiation to DC, the mesodermally differentiated ES cell-derived cells harvested from a 5 or 6-day culture on OP9 feeder layers were cultured on fresh OP9 cell layers in the presence of exogenous GM-CSF. In comparison with the culture without exogenous GM-CSF, addition of this cytokine resulted in appearance of a larger number of floating cells in a few days, indicating that cells grew responding to GM-CSF. Culture without GM-CSF at this step resulted in generation of relatively small numbers of various types of hematopoietic cells (Fig. 2c-e).

On days 8-9 (3 days after the transfer), we observed many round and relatively homogenous floating cells (Fig. 3a), and most of them expressed CD11b, suggesting their commitment to myeloid cell lineage. On day 10 or 11 , we recovered the cells floating or loosely adherent to feeder cells and transferred to bacteriologic Petri dishes without feeder cells. After this passage, some of the transferred cells adhered to the dish surface and resembled macrophages. On days 15-18, floating cells could be divided roughly into 2 types, 1 with a round shape and of a larger size and the other smaller and irregularly shaped with protrusions (Fig. 3b-d). In addition, clusters of floating cells (Fig. 3e) of the latter type were observed after days 


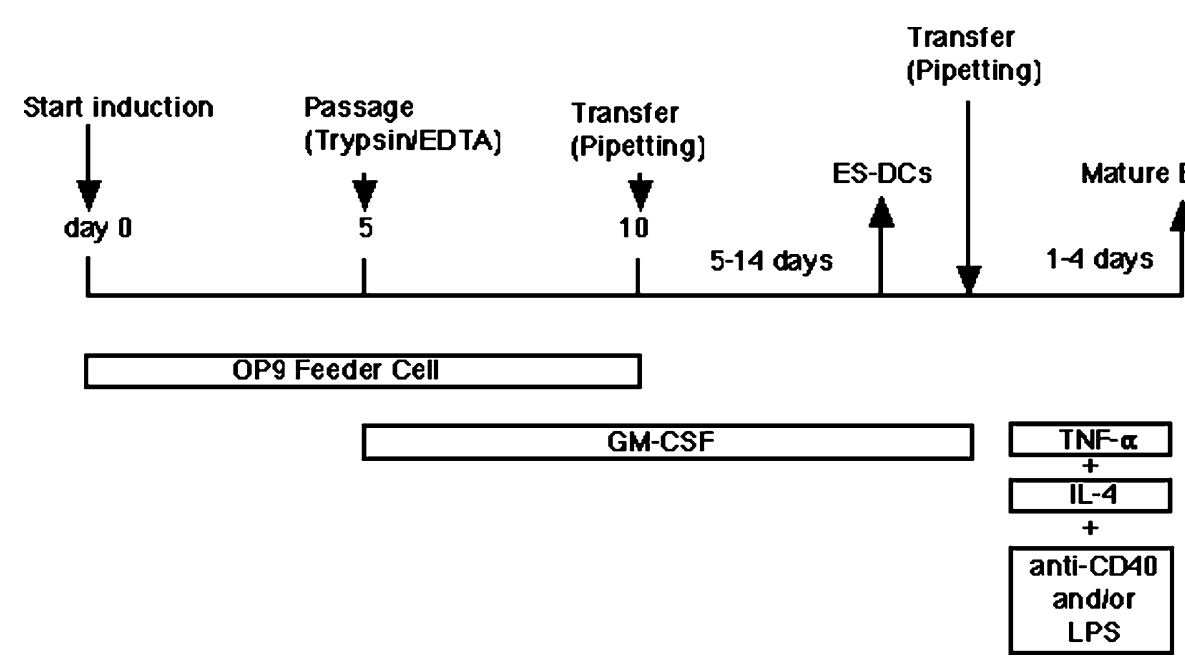

Fig. 1 Schema of the culture protocol for generation of mouse ES-DC. To initiate differentiation, undifferentiated ES cells were suspended in $\alpha$-MEM supplemented with $20 \%$ fetal calf serum (FCS) and seeded onto OP9 cell layers. On days 5-6, cells were harvested using trypsin/EDTA, reseeded onto fresh OP9 cell layers, and cultured in $\alpha$-MEM supplemented with FCS and GM-CSF. 5-6 days after the transfer, floating cells were recovered by pipetting. At this step, 100-200 times more numbers of cells as compared with undifferentiated ES cells was recovered. The recovered cells were transferred to bacteriologic Petri dishes without feeder cells and cultured in RPMI-1640 medium supplemented with $10 \%$ FCS, GM-CSF. After 5-10 days, floating or loosely adherent cells were recovered by pipetting (ES-DC). To induce a complete maturation of ES-DC, cells cultured for longer than 10 days in Petri dishes were transferred to fresh Petri dishes and IL-4, TNF- $\alpha$, plus anti-CD40 $\mathrm{mAb}$, or IL-4, TNF- $\alpha$, plus lipopolysaccharide were added
Fig. 2 Hematopoietic differentiation of TT2 ES cells on feeder cell layers of OP9. Phase-contrast micrographs of TT2 ES cell colonies on OP9 feeder cell layers on day 3 (a) and day 5 (b) are shown. c-e May-Giemsa staining of cytospin specimens of hematopoietic cells derived from TT2 ES cells. TT2 cells were cultured on OP9 feeder cell layer for 15 days in total, without addition of exogenous cytokines. Floating cells were applied to cytospin preparations and stained with May-Giemsa. Cells of myeloid (c), erythroid (d), and megakaryocytic (e) lineages are shown. Scale bars represent $50 \mu \mathrm{m}(\mathbf{a}, \mathbf{b})$ and $20 \mu \mathrm{m}(\mathbf{c}-\mathbf{e})$ (the photo images were originally published in [3])
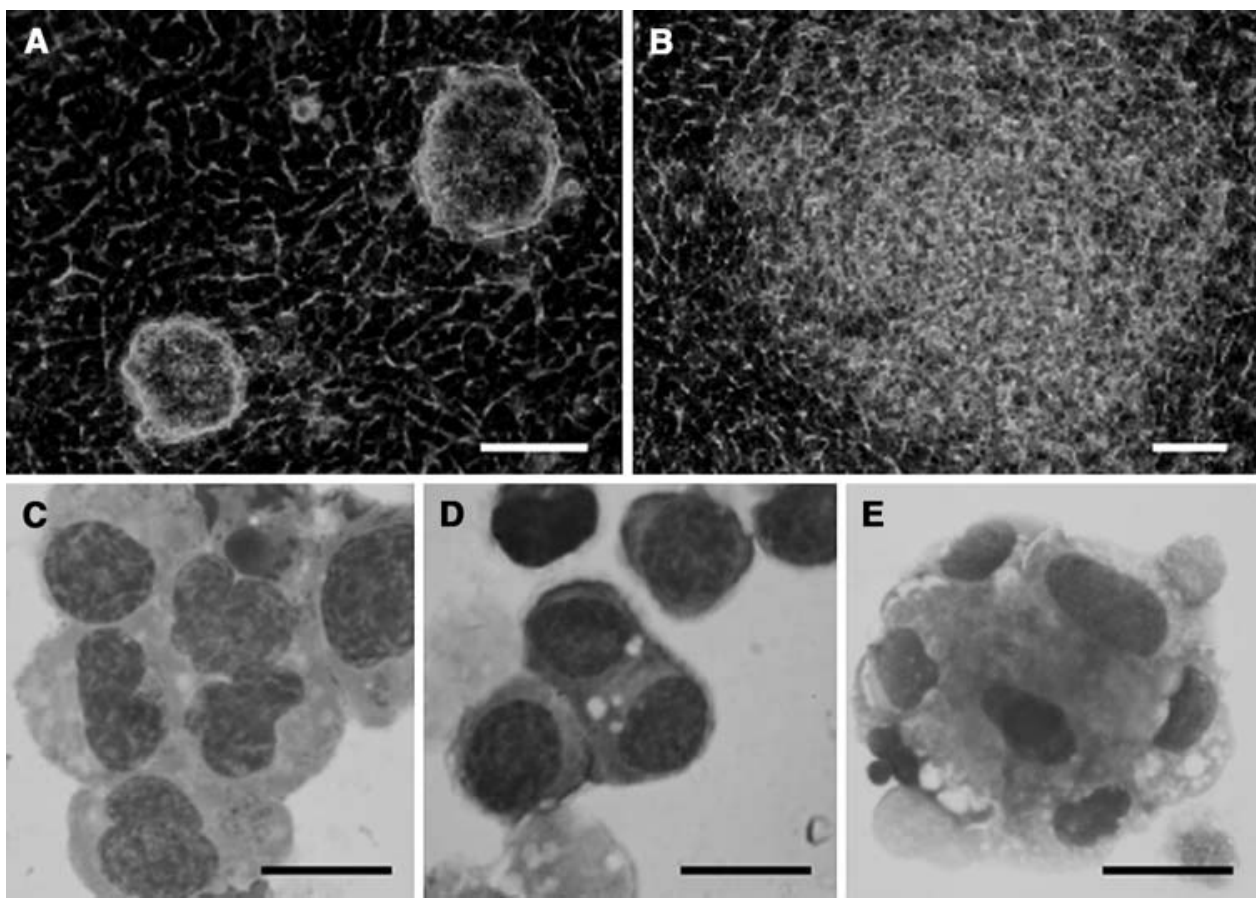

17-19, and the cell clusters gradually increased. Addition of maturation stimuli, IL-4, TNF- $\alpha$, plus anti-CD40 antibody or LPS, to the cells resulted in typical morphology of mature DC (Fig. 3f-h).

Floating cells were positive for MHC class I, MHC class II, CD80, CD86, DEC205, and CD11c. Upon stimulation with LPS they matured and became highly competent to stimulate T cells. Characteristics of the EB-derived esDC seemed to be similar to those of our ES-DC. The cells had capacity to process and present protein antigen to $\mathrm{T}$ cells. They were very potent in stimulating allogeneic $\mathrm{T}$ cells to proliferate (primary mixed lymphocyte reaction). Based on the morphology, surface phenotype, and function, we referred to the cells with protrusions as ES-DC. ES-DC 

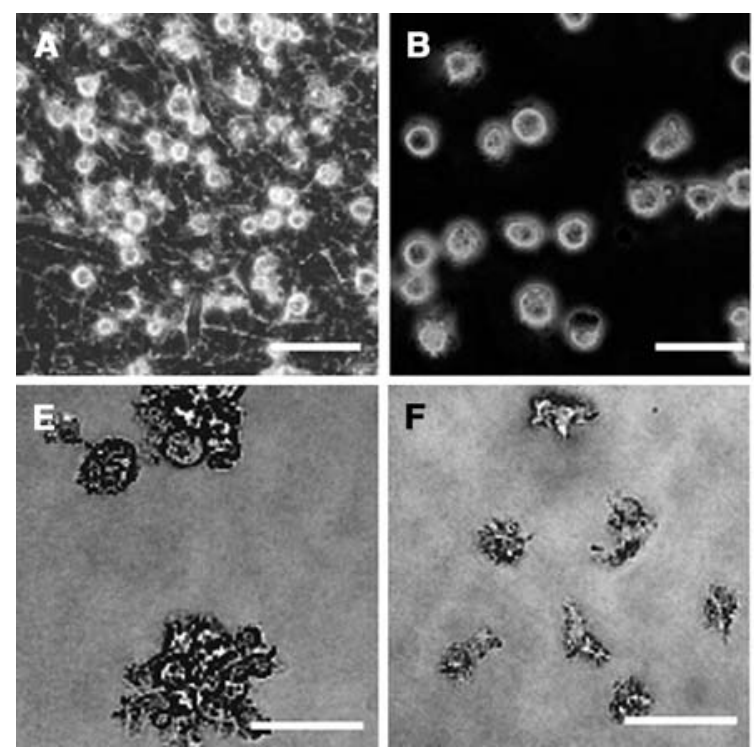

Fig. 3 Morphology of ES-DC. ES cell-derived cells on day 8 (a), day $12(\mathbf{b}, \mathbf{c})$, day 17 (d, e), and day 27 (f) of differentiation culture are shown. Cells on day 24 were recovered and stimulated for 2 days

were positive for $\mathrm{F} 4 / 80$ and $\mathrm{CD} 11 \mathrm{~b}$ and negative for CD8, suggesting that they were of myeloid lineage.

On days 5-10 of the differentiation culture (2nd step), DC could also differentiate when PA6 or ST2 cells were used as feeder cells. However, the number of generated DC was fewer and the phenotype of the generated DC somewhat differed. With PA6 or ST2, generated DC did not express CD80 and CD205, and their activity to stimulate MLR was weaker than that of DC produced with OP9. The use of dishes treated for tissue culture after transfer from the OP9 feeder cell layer (after day 10 in Fig. 1) gave rise to a fewer number and a lower purity of ES-DC than did the use of bacterial-quality Petri dishes. If we used dishes of tissueculture grade, many cells firmly adhered to the dish surface, resembling macrophages or fibroblasts, and inhibited the generation of ES-DC. The beneficial effect of bacterialquality Petri dishes to DC-development has been noted also in generation of DC from mouse bone marrow cells. GMCSF has been reported to be essential for in vitro generation of DC from hematopoietic cells and is also necessary for generation of ES-DC. We applied this culture protocol to various mouse ES cell lines, including TT2, E14, J1, D3, R1, CCE, and NOD mouse-derived ES cells, and observed that all of these lines also differentiated to ES-DC.

\section{Studies on immune-therapy with ES-DC}

\subsection{Priming of antigen-specific cytotoxic $\mathrm{T}$ cells with genetically modified ES-DC}

Genetic modification of ES-DC can readily be done by gene-introduction into undifferentiated ES cells, isolation

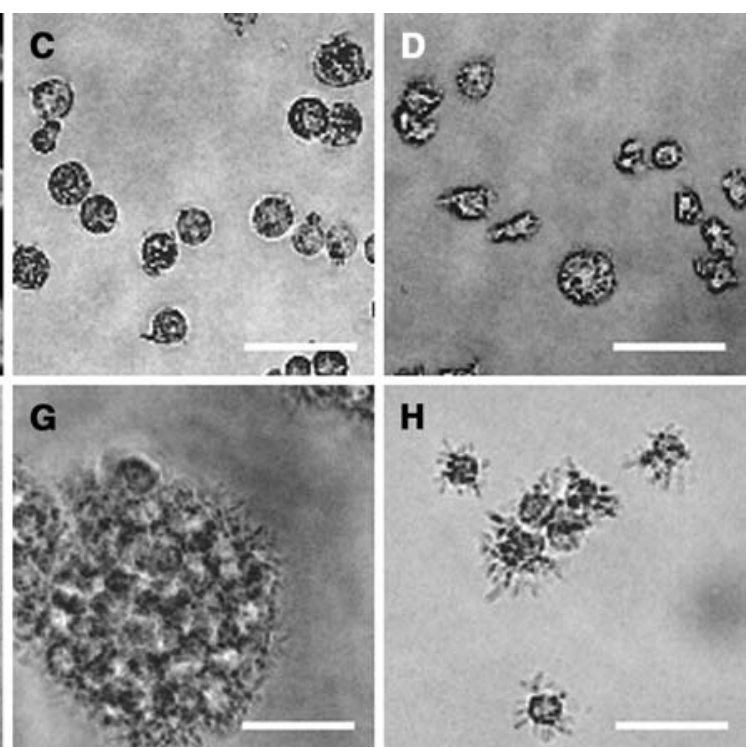

with IL-4, TNF- $\alpha$, plus agonistic anti-CD40 mAb (g), or with IL-4, TNF- $\alpha$, plus LPS (h). a, b Phase-contrast micrographs. Scale bars represent $20 \mu \mathrm{m}$ (the photo images were originally published in [3])

of proper transfectant ES clones, and subsequent induction of differentiation into ES-DC. The capacity of TT2 ES cellderived ES-DC introduced with an ovalbumin (OVA)expression vector to prime OVA-specific $\mathrm{T}$ cells in vivo was analyzed [3]. ES-DC with or without OVA expression vector were injected intraperitoneally into syngeneic $(\mathrm{C} 57 \mathrm{BL} / 6 \times \mathrm{CBA}) \mathrm{F} 1$ mice twice with a 7-day interval. Splenocytes were isolated 7 days after the second injection and cultured in vitro in the presence of OVA257-264 peptide, the major $\mathrm{H}-2 \mathrm{~K}^{\mathrm{b}}$-restricted epitope derived from OVA protein. After 5 days, cultured cells were assayed for their capacity to kill EL-4 thymoma cells $\left(\mathrm{H}-2^{\mathrm{b}}\right)$ prepulsed with the OVA peptide. The results shown in Fig. 4 indicated that cytotoxic $\mathrm{T}$ cells (CTL) specific to the OVA epitope were primed in vivo with ES-DC expressing OVA protein (ES-DC-OVA), but not with ES-DC without OVA expression. These results demonstrate that ES-DC genetically engineered to express an antigenic protein have the capacity to prime antigen-specific CTL in vivo.

\subsection{Enhanced priming of antigen-specific CTL in vivo by ES-DC expressing chemokine along with antigenic protein}

In immunotherapy by using DC, such as anti-cancer immunotherapy, the efficiency of $\mathrm{T}$ cell-priming in vivo by transferred $\mathrm{DC}$ is critical. $\mathrm{T}$ cell-priming in vivo by injected DC should depend on their encounter with $\mathrm{T}$ cells. When exogenous antigen was injected intracutaneously, about $25 \%$ of the DC capturing the antigen migrated to the $\mathrm{T}$ cell area of draining lymph nodes, where they present antigen to prime naive $\mathrm{T}$ cells specific to the antigen. 


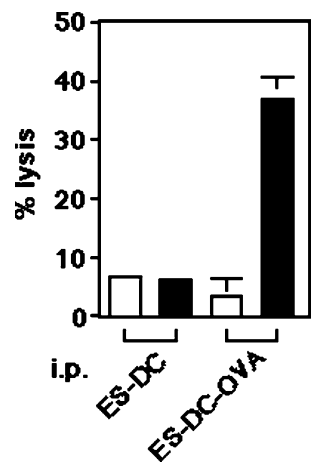

Fig. 4 Priming of OVA-specific CTL by ES-DC expressing OVA in vivo. ES-DC with (ES-DC-OVA) or without (ES-DC) expression of OVA protein were injected intraperitoneally on days 0 and 7 into syngeneic F1 mice. Splenocytes from the injected mice were harvested on day 14 and cultured in the presence of OVA257-264 $(0.1 \mu \mathrm{M})$ for 5 days. The resultant cells were assayed for the capacity to lyse EL-4 tumor cells either pulsed with $10 \mu \mathrm{M}$ OVA peptide (closed bars) or left unpulsed (open bars)

On the contrary, when BM-DC or splenic DC are transferred exogenously by s.c. or i.p. injection, the absolute number of the DC found within the draining lymph nodes represented only a small proportion $(0.1-1 \%)$. It has been also reported that almost all of transferred DC remained at the s.c. immunization site $24 \mathrm{~h}$ after transfer. The inefficient migration of the exogenous DC to lymphoid organs may lower the frequency of their encounter with $\mathrm{T}$ cells. It may be possible to improve the efficacy of exogenously transferred DC to prime immune responses by augmenting their encounter with T cells. For example, if transferred DC produce chemokines to intensively attract $\mathrm{T}$ cells, they may prime immune response efficiently, even though the DC do not migrate to lymphoid organs. We attempted to improve the capacity of DC to prime $\mathrm{T}$ cells in vivo by genetic modification to express chemokine with $\mathrm{T}$ cell-attracting property. We generated double-transfectant DC expressing a chemokine along with the model antigen, OVA, by sequential transfection of ES cells with 2 kinds of expression vectors and then inducing differentiation to DC [4].

Several kinds of chemokines with capacity to attract $\mathrm{T}$ cells are produced by different cell types. CCL21 (secondary lymphoid tissue chemokine, SLC) is produced in $\mathrm{T}$ cell regions of lymph nodes and spleen and also by high endothelial venules in lymph nodes. SLC chemoattracts T cells, NK cells, B cells, and DC. CXCL9 (monokine induced by IFN- $\gamma$, Mig) is produced by macrophages and binds to the chemokine receptor CXCR3, which mediates the recruitment of predominantly Th1 cells and activated NK cells. XCL1 (Lymphotactin, Lptn), produced by activated $\mathrm{T}$ cells, has chemoattractive properties on $\mathrm{CD}^{+}$and $\mathrm{CD}^{+} \mathrm{T}$ cells and on NK cells. We comparatively evaluated the effect of these 3 kinds of chemokines.
All three types of double transfectant ES-DC primed OVA-specific CTL in vivo more efficiently than did ESDC expressing only OVA, and co-expression of SLC or Lptn was more effective than that of Mig. Immunization with DC expressing OVA plus SLC or Mig provided protection from OVA-expressing tumor cells more potently than that with OVA only, and SLC was more effective than Mig. On the other hand, co-expression of Lptn gave no additive effect on protection from the tumor. collectively, among the three chemokines, expression of SLC was the most effective in enhancing anti-tumor immunity by transferred ES-DC in vivo.

\subsection{Negative manipulation of immune response} for the treatment of autoimmune disease

For the treatment of subjects with autoimmune or inflammatory diseases, it is desirable to down-modulate immune responses in an antigen-specific manner and without causing systemic immune suppression. GVHD and graft rejection are the most serious problems in transplantation medicine, and control of allo-reactive immune response is the key to overcome them. Antigen-specific negative regulation by DC is considered to be promising means in these fields. We investigated whether we could negatively manipulate immune response with genetically modified ES-DC expressing specific antigen along with immunesuppressive molecules.

Experimental autoimmune encephalomyelitis (EAE), characterized by neurological impairment resulting from de-myelination in the central nervous system caused by activated myelin antigen-reactive $\mathrm{CD} 4^{+} \mathrm{T}$ cells, is induced by immunization with myelin antigens such as myelin oligodendrocyte glycoprotein (MOG) or myelin basic protein (MBP). We tried to prevent MOG-induced EAE by pre-treatment of mice with genetically modified DC presenting MOG peptide in the context of MHC class II molecules and simultaneously expressing molecules with $\mathrm{T}$ cell-suppressive property [10].

For efficient presentation of MOG peptide in the context of MHC class II molecules, we used a previously devised expression vector in which cDNA for human MHC class IIassociated invariant chain (Ii) was mutated to contain antigenic peptide in the class II-associated invariant chain peptide (CLIP) region. An epitope inserted in this vector is efficiently presented in the context of co-expressed MHC class II molecules [13].

As T cell-suppressive molecules, we tested TNF-related apoptosis-inducing ligand (TRAIL) and programmed death-1 ligand (PD-L1). TRAIL as a member of the TNF superfamily is constitutively expressed in a variety of cell types, including lymphocytes, NK cells, and neural cells. TRAIL $^{-/-}$mice are hypersensitive to collagen-induced 
arthritis and streptozotocin-induced diabetes. PD-L1, a ligand for PD-1 and member of the CD28/CTLA-4 family, is expressed on DC, IFN- $\gamma$-treated monocytes, activated $\mathrm{T}$ cells, placental trophoblasts, myocardial endothelium, and cortical thymic epithelial cells. PD- $1^{-/-}$mice spontaneously develop a lymphoproliferative/autoimmune disease, a lupus-like disease, arthritis, and cardiomyopathy [14]. Thus, abrogation of either of these two molecules makes mice autoimmune-prone, suggesting that these molecules play significant roles in maintaining immunological selftolerance in physiological situations.

ES cells were sequentially transfected with an expression vector for TRAIL or PD-L1 and an MHC class II-associated invariant chain-based MOG epitope presenting vector. Subsequently, double-transfectant ES cell clones were induced to differentiate into ES-DC expressing the introduced genes, ES-DC-TRAIL/MOG, and PDL1/MOG.

Severity of MOG peptide-induced EAE was significantly reduced in mice pre- or post-treated with ES-DCTRAIL/MOG or ES-DC-PDL1/MOG. The severity of MBP-induced EAE was also reduced by ES-DC-TRAIL/ MOG but not PD-L1/MOG. In addition, adoptive transfer of $\mathrm{CD}^{+}{ }^{+} \mathrm{T}$ cells from ES-DC-TRAIL/MOG-treated mice protected the recipient mice from subsequent induction of EAE [11]. These results demonstrate the prevention of an autoimmune disease by the treatment with ES-DC expressing target antigen along with immune-suppressive molecules, and implicate future clinical application of this technology.

\section{Generation of DC from human ES cells}

\subsection{Generation of DC from human ES cells based on EB formation}

As for the generation of DC from human ES cells, the report by Zhan and colleagues [15] is the first. Similar to the method of generation of DC from mouse ES cells by Fairchild and colleagues, they induced formation of EB by suspension culture of human ES cells, and then cultured the $\mathrm{EB}$ in the presence of hematopoietic cytokines to generate leukocytes with antigen presenting function.

In their method, human ES cells were cultured at a highdensity onto Matrigel (Becton Dickson) before induction of differentiation. After reaching the full size or confluency, undifferentiated ES cell colonies were harvested with dispase. The cell colonies were cultured in medium for differentiation induction with fetal calf serum and without basic fibroblast growth factor (bFGF). To inhibit the adherence to the plastic surface of the culture plates, ES colonies were plated in special low-attachment plates. Cystic embryonic bodies emerged after 5-20 days in the suspension culture. When harvested on days 10-20, $50-80 \%$ of EB were cystic, each EB containing about 10,000 cells on average.

Whole EB formed in suspension were transferred onto tissue culture plates and allowed to differentiate into hemopoietic and other cell types. To stimulate production of hemopoietic progenitor cells and also DC, they added stem cell factor (SCF), FLT3-ligand, and thrombopoietin (TPO), cytokines widely used to maintain human postnatal haemopoietic stem cells. To expand DC-committed progenitor cells IL-3, GM-CSF, and IL-4 were added.

A part of the resulting leucocytes expressed MHC class II, CD80, CD86, CD40, and CD83. The MHC class $\mathrm{II}^{+}$ leucocytes resembled DC and macrophages. They functioned as stimulator cells capable of eliciting allogeneic $\mathrm{T}$ cell responses in culture.

After this report, Su and colleagues [16] and also Tseng and colleagues [17] reported similar methods of EB-mediated generation of DC from human ES cells.

\subsection{Generation of human ES-DC based on OP9-co-culture}

Considering future clinical application of ES-DC, we also tried to generate ES-DC from human ES cells [12]. Based on experience in the generation of DC from mouse ES cells and also based on the findings in a preliminary study using cynomolgus monkey ES cells [18], feeder cell-co-culture method was adopted for the generation of DC from human ES cells, instead of the EB based method. The human ES cell line selected was KhES-1 which exhibited the highest growth rate and stability among the 3 lines of human ES cell lines established by Suemori and colleagues [19] at Kyoto University. For feeder cells, 3 lines of mouse stromal cell lines, ST2, OP9, and PA6 were evaluated for their capacity to induce hematopoietic differentiation of KhES-1 ES cells, and OP9 had the best yield among them.

The protocol for the differentiation culture to generate ES-DC from human ES cells developed in our study is composed of 3 steps. At the beginning of the differentiation culture, undifferentiated ES cells maintained on mouse PEF feeders were harvested using dissociation solution, CTK, and plated on OP9 feeder cell layers (step 1). Next, the ES cells grew and formed clusters composed mostly of epithelial cell-like large flat cells. Clusters of round, cobble stone-like cells also appeared on about day 8 . The size and number of round cell clusters gradually increased, and, around day 15 , covered $20-30 \%$ of the surface area.

On days $15-18$ of the step 1 culture, cells were recovered from the dishes using trypsin/EDTA and isolated nonadherent cells, and then they were seeded onto freshly prepared OP9 cell layers, to begin step 2. Culture medium containing GM-CSF and M-CSF was used in this step. 
Thereafter, small round cells, floating or loosely adhering to the feeder layer, appeared and gradually increased in number. The growth of the round cells depended primarily upon GM-CSF, thus suggesting that they grew in response to that factor. The cells expressed CD34 and CD45, thus indicating that they followed a hematopoietic cell lineage. They also expressed CD31, CD43, and CD11b, thus collectively indicating a commitment to a myeloid cell lineage.

On days $7-10$ of the step 2 culture the floating or loosely adherent cells were harvested by pipetting, and they were transferred to Petri dishes without feeder cells and cultured in the presence of GM-CSF and IL-4 (step 3). Following this passage, the cells changed their morphology from round to irregular shape, and some had protrusions. Cells with protrusions gradually increased and more than $50 \%$ of the cells exhibited DC-like irregular shape after 2-3 days. The floating cells expressed CD86 and CD40, but scarcely expressed CD80 and CD83.

Simultaneous addition of TNF- $\alpha$, LPS, soluble CD40ligand, and IL-4 to the cells induced maturation. Many of the cells formed aggregates. Flowcytometric analysis showed the increased expression of CD86 and the expression of CD80, CD83, and HLA-DR. Collectively, the cells exhibited the characteristics of DC in their morphology and expression of surface molecules, and thus they were designated as human ES-DC. Production of TNF- $\alpha$ was profoundly induced by either LPS or OK432. OK432, but LPS did not induce the production of IL-12, consistent with the reports that OK432 is an efficient inducer of IL-12.

ES-cell-derived floating cells first appeared during the 2nd step of the culture for differentiation (pre-ES-DC) and could readily be isolated by pipetting procedure. Their morphology, pattern of expression of surface molecules, and $\mathrm{T}$ cell-stimulation capacity continuously changed until the final maturation. To determine the change in gene expression associated with such changes in the phenotypes, the gene expression profiles of pre-ES-DC, immature ESDC, and mature ES-DC were analyzed using cDNA microarrays. Consistent with the results of flowcytometric analysis, up-regulation of the expression of genes encoding for cell surface molecules such as HLA class I, HLA class II, CD86, and CD40 along with differentiation of ES-DC was observed. In addition, expression of the genes related to DC function including CD74/invariant chain, CCR7, and CCL17/TARC was increased during the differentiation. Clustering analysis indicates similarity between change of the gene expression pattern from monocytes to immature Mo-DC and that from pre-ES-DC to immature ES-DC as well as that from immature Mo-DC to mature Mo-DC and that from immature ES-DC to mature ES-DC.

The protocol of differentiation culture described to this point was originally developed using the KhES- 1 line of human ES cells. This differentiation procedure was also applied to KhES-3, another human ES cell line. KhES-3 differentiation was similar to KhES-1 except that KhES-3 differentiated slightly more quickly than KhES-1, and a first step culture of 14-15 days was sufficient for the differentiation of KhES-3. Generation of ES-DC from nonhuman primate, cynomolgus monkey, ES cells was also achieved by this method [12].

Slukvin and colleagues [20] also reported a method using OP9. Slukvin's method is also composed of 3 steps and is different from ours in that OP9 feeders were used only in the first step. Recently, extending this study, they demonstrated generation of various myelomonocytic cells including DC, macrophages, osteoclasts, neutrophils, and eosinophils from human ES cells or induced pluripotent stem (iPS) cells [21].

\subsection{Function of human ES-DC as antigen presenting cells}

The capacity of the human ES-DC to stimulate T cells was examined based on the proliferative response of allogeneic $\mathrm{T}$ cells co-cultured with ES-DC. ES cell-derived floating cells recovered from the second step (pre-ES-DC) showed little capacity to induce a response of $\mathrm{T}$ cells. In contrast, ES-DC following the third step before the addition of maturation stimuli (immature ES-DC) showed a weak but definite stimulation, and following exposure to the maturation stimuli (mature ES-DC) showed a strong capacity to stimulate allogeneic $\mathrm{T}$ cells to proliferate.

Next, the antigen-presenting capacity of ES-DC was examined. KhES- 1 is positive for the HLA-DRB $4 * 0103$ gene encoding $\beta$ chain of HLA-DR53 molecule. Presumably, ES-DC derived from KhES-1 should express the DR53 molecule, and their ability to present antigen to DR53-restricted $\mathrm{CD}^{+}{ }^{+} \mathrm{T}$ cells was determined. KhES-1derived ES-DC pre-loaded with GAD65-derived synthetic peptide stimulated GAD65-specific DR53-restricted human $\mathrm{T}$ cell clone, SA32.5 [22], to proliferate. To examine the capacity to process antigenic protein and present epitope, recombinant protein was used as the antigen. The SA32.5 $\mathrm{T}$ cell clone co-cultured with the ES-DC in the presence of recombinant GAD65 protein also showed proliferative response, thus indicating that ES-DC processed the antigenic protein and presented the epitope derived from the protein in the context of HLA class II molecules.

\subsection{Genetic modification of human ES-DC}

We applied the strategy for the genetic modification of ESDC established by using mouse ES cells to human ES cells. Briefly, the expression vectors were introduced into ES 
cells by electroporation and subsequently the transfectant ES cell clones were induced to differentiate to ES-DC.

ES-DC carrying an epitope-presenting vector and expressing recombinant human invariant chain (Ii/CD74), which included GAD65p115-127 in the CLIP region, was generated. It was expected that the epitope could be efficiently targeted on MHC class II pathway [13]. The vector was introduced into KhES-1 ES cells, and a transfectant clone, KhES-1-Ii23, highly expressing transgene-derived recombinant CD74 was selected by a flowcytometric analysis at the pre-ES-DC stage. The expression of CD74 was detected even in the non-transfectant pre-ES-DC, reflecting intrinsic expression of CD74. The transfectant exhibited an increased expression of CD74 in comparison with the non-transfectants, thus indicating additional expression of the molecule derived from the transgene. The ability of the transfectant ES-DC, ES-DC-Ii23 to stimulate the GAD-epitope-specific $\mathrm{T}$ cell clone, SA32.5, in the absence of antigenic peptide or protein was next examined. As a result, ES-DC-Ii23 stimulated SA32.5 T cells and induced their proliferation, thus demonstrating functional expression of the epitope-presentation vector in the transfectant ES-DC. The in vivo transfer of ES-DC transfected with this antigen-presenting vector is therefore expected to be useful for controlling the immune response in an antigen-specific manner.

$\mathrm{Su}$ and colleagues [16] introduced mRNA for green fluorescence protein (GFP) or prostate-specific antigen (PSA) into DC generated from human ES cells. They showed induction of CTL specific to the antigens by in vitro stimulation of semi-allogeneic, HLA-A*0201 ${ }^{+}$, donor-derived $\mathrm{T}$ cells with the genetically modified DC.

\section{Generation of DC from mouse iPS cells}

As described so far, we can generate ES-DC from both mouse and human ES cells. Mouse systems have demonstrated the induction of anti-cancer immunity and the prevention of autoimmune disease by in vivo administration of genetically engineered ES-DC.

Considering the future clinical application of ES-DC technology, however, the unavailability of human ES cells genetically identical to the patients to be treated is a problem. Allogenicity caused by differences in the genetic background between human ES cell lines and the recipients is a critical problem in medical application of ES-DC. In addition, ethical concerns related to the use of human ES cells are anticipated to be serious obstacles, which will hinder the realization of the medical use of human ES-DC.

It was recently revealed that ES cell-like pluripotent stem cells, designated as iPS cells, can be generated by the simultaneous introduction of several genes for re-programming factors, such as Oct3/4, Sox2, Klf4, and c-Myc, into somatic cells, for both mouse and human [2325]. The issue of histoincompatibility between patients to be treated and ES cells may be overcome by the generation of iPS cells from somatic cells of the patients such as fibroblasts or blood cells. The major ethical issues related to human ES cells would be avoided by aid of iPS celltechnology, because the use of human embryos is not necessary for the generation of iPS cells.

Differentiation of iPS cells into various cells belonging to the 3 germ layers has been demonstrated by the analysis of teratomas generated from mouse and human iPS cells. In addition, the pluripotency of iPS cells is obvious by the contribution of iPS cell-derived cells to various organs of the chimeric mice developed from iPS cell-introduced blastocysts. As for the in vitro generation of cells of mesodermal lineage from iPS cells, differentiation into cardiac myocytes and endothelial cells from mouse iPS cells has been reported.

We recently reported the generation and characterization of DC derived from mouse iPS cells [26]. The iPS cellderived DC (iPS-DC) possessed the characteristics of DC including the capacity of $\mathrm{T}$ cell-stimulation, antigen-processing and presentation, and cytokine production. DNA microarray analyses revealed the up-regulation of genes related to antigen-presenting functions during differentiation into iPS-DC and similarity in gene-expression profile in iPS-DC and bone marrow cell-derived DC. There was some delay in the kinetics of differentiation process of iPS cells, as in comparison with most of mouse ES cell lines. On the other hand, the yield of differentiated cells was higher than that in the cases of most of mouse ES cell lines. Genetically modified iPS-DC expressing antigenic protein primed $\mathrm{T}$ cells specific to the antigen in vivo and elicited efficient antigen-specific anti-tumor immunity.

\section{Future direction}

We recently succeeded in generating iPS-DC from human iPS cells. Human iPS-DC exhibited characteristics of DC, as human ES-DC do, in morphology, surface molecules, and $\mathrm{T}$ cell-stimulating capacity. Theoretically, we can now generate infinite number of DC genetically matched to the patients by using iPS cells generated from relatively small number of somatic cells of the patients, such as dermal fibroblasts or blood cells.

Considering clinical application of iPS-DC, the technical issue to be resolved is that we are still using mousederived OP9 feeder cells and culture medium containing fetal calf serum for the differentiation culture. A xeno-free culture method to maintain human iPS cells has been already developed, and we should make considerable effort 
to establish xeno-free differentiation culture. Other issues to be considered may be the time and cost for production of iPS cells specific to the individual patients. This is not the issue specific to iPS-DC therapy, and in this regard, establishment of a bank of iPS cells derived from somatic cells of HLA haplotype-homozygous donors would be profoundly valuable [27].

\section{References}

1. Nakano T, Kodama H, Honjo T. Generation of lymphohematopoietic cells from embryonic stem cells in culture. Science. 1994;265:1098-101.

2. Fairchild PJ, Brook FA, Gardner RL, et al. Directed differentiation of dendritic cells from mouse embryonic stem cells. Curr Biol. 2000;10:1515-8.

3. Senju S, Hirata S, Matsuyoshi $\mathrm{H}$, et al. Generation and genetic modification of dendritic cells derived from mouse embryonic stem cells. Blood. 2003;101:3501-8.

4. Matsuyoshi H, Senju S, Hirata S, et al. Enhanced priming of antigen-specific CTLs in vivo by embryonic stem cell-derived dendritic cells expressing chemokine along with antigenic protein: application to antitumor vaccination. J Immunol. 2004;172:776-86.

5. Matsuyoshi H, Hirata S, Yoshitake Y, et al. Therapeutic effect of alpha-galactosylceramide-loaded dendritic cells genetically engineered to express SLC/CCL21 along with tumor antigen against peritoneally disseminated tumor cells. Cancer Sci. 2005;96:889-96.

6. Fukuma D, Matsuyoshi H, Hirata S, et al. Cancer prevention with semi-allogeneic ES cell-derived dendritic cells. Biochem Biophys Res Commun. 2005;335:5-13.

7. Motomura Y, Senju S, Nakatsura T, et al. Embryonic stem cell-derived dendritic cells expressing glypican-3, a recently identified oncofetal antigen, induce protective immunity against highly metastatic mouse melanoma, B16-F10. Cancer Res. 2006;66:2414-22.

8. Fukushima S, Hirata S, Motomura Y, et al. Multiple antigentargeted immunotherapy with alpha-galactosylceramide-loaded and genetically engineered dendritic cells derived from embryonic stem cells. J Immunother. 2009;32:219-31.

9. Matsunaga Y, Fukuma D, Hirata S, et al. Activation of antigenspecific cytotoxic $\mathrm{T}$ lymphocytes by beta 2 -microglobulin or TAP1 gene disruption and the introduction of recipient-matched MHC class I gene in allogeneic embryonic stem cell-derived dendritic cells. J Immunol. 2008;181:6635-43.

10. Hirata S, Senju S, Matsuyoshi H, et al. Prevention of experimental autoimmune encephalomyelitis by transfer of embryonic stem cell-derived dendritic cells expressing myelin oligodendrocyte glycoprotein peptide along with TRAIL or programmed death-1 ligand. J Immunol. 2005;174:1888-97.
11. Hirata S, Matsuyoshi H, Fukuma D, et al. Involvement of regulatory $\mathrm{T}$ cells in the experimental autoimmune encephalomyelitis-preventive effect of dendritic cells expressing myelin oligodendrocyte glycoprotein plus TRAIL. J Immunol. 2007;178:918-25.

12. Senju S, Suemori H, Zembutsu H, et al. Genetically manipulated human embryonic stem cell-derived dendritic cells with immune regulatory function. Stem Cells. 2007;25:2720-9.

13. Fujii S, Senju S, Chen YZ, et al. The CLIP-substituted invariant chain efficiently targets an antigenic peptide to HLA class II pathway in L cells. Hum Immunol. 1998;59:607-14.

14. Okazaki T, Honjo T. The PD-1-PD-L pathway in immunological tolerance. Trends Immunol. 2006;27:195-201.

15. Zhan X, Dravid G, Ye Z, et al. Functional antigen-presenting leucocytes derived from human embryonic stem cells in vitro. Lancet. 2004;364:163-71.

16. Su Z, Frye C, Bae KM, et al. Differentiation of human embryonic stem cells into immunostimulatory dendritic cells under feederfree culture conditions. Clin Cancer Res. 2008;14:6207-17.

17. Tseng SY, Nishimoto KP, Silk KM, et al. Generation of immunogenic dendritic cells from human embryonic stem cells without serum and feeder cells. Regen Med. 2009;4:513-26.

18. Suemori H, Tada T, Torii R, et al. Establishment of embryonic stem cell lines from cynomolgus monkey blastocysts produced by IVF or ICSI. Dev Dyn. 2001;222:273-9.

19. Suemori H, Yasuchika K, Hasegawa K, et al. Efficient establishment of human embryonic stem cell lines and long-term maintenance with stable karyotype by enzymatic bulk passage. Biochem Biophys Res Commun. 2006;345:926-32.

20. Slukvin II, Vodyanik MA, Thomson JA, et al. Directed differentiation of human embryonic stem cells into functional dendritic cells through the myeloid pathway. J Immunol. 2006;176:2924-32.

21. Choi KD, Vodyanik MA, Slukvin II. Generation of mature human myelomonocytic cells through expansion and differentiation of pluripotent stem cell-derived lin-CD34+CD43+CD45+progenitors. J Clin Invest. 2009;119:2818-29.

22. Tabata H, Kanai T, Yoshizumi H, et al. Characterization of selfglutamic acid decarboxylase 65-reactive CD4+ T-cell clones established from Japanese patients with insulin-dependent diabetes mellitus. Hum Immunol. 1998;59:549-60.

23. Takahashi K, Yamanaka S. Induction of pluripotent stem cells from mouse embryonic and adult fibroblast cultures by defined factors. Cell. 2006;126:663-76.

24. Okita K, Ichisaka T, Yamanaka S. Generation of germline-competent induced pluripotent stem cells. Nature. 2007;448:313-7.

25. Takahashi K, Tanabe K, Ohnuki M, et al. Induction of pluripotent stem cells from adult human fibroblasts by defined factors. Cell. 2007;131:861-72.

26. Senju S, Haruta M, Matsunaga $Y$, et al. Characterization of dendritic cells and macrophages generated by directed differentiation from mouse induced pluripotent stem cells. Stem Cells. 2009;27:1021-31.

27. Nakatsuji N, Nakajima F, Tokunaga K. HLA-haplotype banking and iPS cells. Nat Biotechnol. 2008;26:739-40. 